Universidade Tecnológica Federal do Paraná - UTFPR

Campus Ponta Grossa - Paraná - Brasil

ISSN: 1981-3686 / v. 03, n. 02: p. 97-108, 2009
Revista Brasileira deTecnologia

Agroindustrial

\title{
CARACTERIZAÇÃO FÍSICA E QUÍMICA E PERFIL LIPÍDICO DE TRÊS ESPÉCIES DE PEIXES AMAZÔNICOS
}

\section{PHYSICAL AND CHEMICAL CHARACTERIZATION AND LIPID PROFILE OF THREE AMAZON FISH SPECIES}

\author{
Ádria de Sousa Bentes ${ }^{1}$; Hugo Antonio Lima de Souza ${ }^{2}$ : Xaene Maria Fernandes Duarte Mendonça ${ }^{3}$ \\ Marilda Garcia Simões ${ }^{4}$; \\ 1,2,3 Universidade Federal do Pará - UFPA - Belém - Brasil adriabentes@ufpa.br \\ ${ }^{4}$ Universidade do Estado do Pará - UEPA - Belém - Brasil marildags@uol.com.br
}

\begin{abstract}
Resumo
O estado do Pará é o segundo maior produtor de pescado do Brasil, no entanto, as informações nutricionais sobre as espécies de peixes amazônicos são escassas. Neste estudo, objetivou-se caracterizar fisicamente e quimicamente, determinar o perfil lipídico e avaliar a qualidade nutricional das espécies: Gurijuba (Arius parkeri), Piramutaba (Brachyplastystoma vaillantii) $e$ Dourada (Brachyplastystoma flavicans). Os modelos matemáticos do peso em função do comprimento e da circunferência das espécies mostraram-se adequados $\left(R^{2}>0,89\right)$. A Gurijuba apresentou o maior teor de cinzas (1,12\%) dentre as espécies. A Piramutaba apresentou o maior teor de proteínas $(18,47 \%)$ e a Dourada, o maior teor de lipídios $(0,53 \%)$, ambos em relação à Gurijuba; não houve diferença entre a piramutaba e a dourada nestes dois parâmetros. $O$ ácido graxo predominante nas três espécies foi o palmítico (27,51 a 29,14\%), seguido do DHA (15,54 a 21,54\%) e do EPA (8,71 a 14,27\%). A Piramutaba apresentou o menor somatório de ácidos graxos $\omega 3(29,8 \%)$. Não foram detectados ácidos graxos $\omega 6$ na Gurijuba nem na Piramutaba. As recomendações da American Heart Association para EPA + DHA são atendidas pelas seguintes porções de filé cru: $248 \mathrm{~g}$ de Gurijuba, $234 \mathrm{~g}$ de Piramutaba e $187 \mathrm{~g}$ de Dourada. A Dourada apresentou excelente ratio $\Sigma A G \omega 6 / \Sigma A G \omega 3$ (1:50). Todas as espécies apresentaram elevada qualidade nutricional da fração lipídica, traduzida por baixos índices IA e IT e elevados HH e ratio $\Sigma P U F A s / \Sigma S F A s$.
\end{abstract}

Palavras-chave: peixes amazônicos; perfil lipídico; qualidade nutricional

\section{Introdução}

Os ácidos graxos da família n-3 tem sido amplamente estudados em virtude de a sua ingestão estar associada a diversos benefícios à saúde humana, como redução dos níveis de depressão durante a gravidez (GOLDING et al., 2009); desaceleração do declínio dos domínios cerebrais relacionados a velocidade cognitiva com o avanço da idade (DULLEMEIJER et al., 2007); e efeito hipocoagulante, independente da vitamina K (VANSCHOONBEEK et al, 2004). 
Segundo Weaver et al. (2009) os benefícios associados a ingestão de ácidos graxos polinsaturados (PUFA) são proporcionados pela sua capacidade de regular a expressão dos genes para citocinas pró-inflamatórias. Dietas ricas em ácidos graxos polinsaturados (PUFA) estão associadas à diminuição dos sintomas de esquizofrenia (MOSTOFSKY; YEHUDA; SALEM, 2001).

A partir dos PUFAs derivam substâncias denominadas eicosanóides, que são hormônios parácrinos, isto é, atuam somente em células próximas ao local onde foram sintetizados por um curto período, devido a sua rápida degradação. Estes compostos são produzidos em quantidades muito pequenas, mas que apresentam funções metabólicas potentes, como regulação da pressão sanguínea, diurese, agregação plaquetária, sistema imune, secreções gástricas, reprodução, contração de músculos lisos, etc. A ingestão excessiva de PUFAs $\omega 6$ está associada ao aumento da produção eicosanóides que potencializam a inflamação (LEHMAN, 2001; LANDS, 2005; MOFFATT; STAMFORD, 2005; NELSON; COX, 2005; SACKHEIM; MOLNAR, 2006).

Uma das fontes de PUFAs n-3 mais citadas na literatura são os peixes, e a sua ingestão regular tem sido associada à redução nos níveis séricos dos mediadores responsáveis pela inflamação e pela resistência à insulina (LANKINEN et al., 2009). Outros estudos mostram ainda, que o consumo moderado de peixe ou óleo de peixe $(\approx 250 \mathrm{mg}$ de EPA + DHA $)$ reduz os riscos de doença cardíaca aguda e crônica (ISO et al., 2006; MOZAFFARIAN, 2008).

Em 2006 a produção de pescado estimada no Brasil foi de 1.050.808 toneladas. Deste total, o Pará foi responsável por 14,5\%, o que o colocou como o segundo estado que mais produziu pescado no Brasil, ficando atrás somente do estado de Santa Carina (15,7\%). E com relação às espécies mais capturadas, a Piramutaba (Brachyplastystoma vaillantii) e a Dourada (Brachyplastystoma flavicans) destacam-se como as mais capturadas na pesca continental e a Gurijuba (Arius parkeri) é a $4^{\mathrm{a}}$ espécie mais capturada na pesca marinha no Pará (BRASIL, 2008).

Assim, levando-se em consideração a importância do pescado no mercado, bem como o seu potencial nutritivo, associado à escassez de informações referentes à composição dos peixes amazônicos, este trabalho objetivou avaliar as características físicas (peso, comprimento zoológico e circunferência abdominal), químicas (umidade, resíduo mineral fixo, proteína bruta e lipídios totais) e determinar o perfil de ácidos graxos - saturados, monoinsaturados e poliinsaturados das séries n-3 e n-6 - de três espécies de peixes amazônicos: Gurijuba (Arius parkeri), Piramutaba (Brachyplastystoma vaillantii) e Dourada (Brachyplastystoma flavicans). E avaliar ainda a qualidade nutricional da fração lipídica dos peixes estudados, através dos índices de aterogenicidade, trombogenicidade e razão entre ácidos graxos hipocolesterolêmicos e hipercolesterolêmicos. 


\section{Materiais e Métodos}

A coleta dos peixes foi realizada no mercado do Ver-o-Peso, município de Belém (PA). A coleta dos peixes foi feita no período de julho a dezembro (época da seca). O estudo não considerou sexo, idade ou estágio de maturação dos peixes.

\subsection{Preparo das amostras}

Para cada espécie foram selecionados aleatoriamente 20 unidades de cada espécie, e posterior agrupamento aleatório em 4 lotes de 5 peixes. Análise dos parâmetros de frescor foi efetuada de acordo com Larsen (1991).

\subsection{Caracterização física}

A caracterização física foi realizada através das medidas: do peso; do comprimento zoológico (medida que vai desde o extremo anterior da cabeça até os raios medianos da nadadeira caudal, estando o animal estendido sobre uma superfície plana); e da circunferência corporal (medida na região imediatamente posterior ao fim da primeira nadadeira dorsal).

\subsection{Caracterização química}

Para as análises químicas, executou-se o seguinte procedimento para cada espécie: extraiuse uma porção da musculatura da parte superior de um dos filés de cada exemplar, e em seguida homogeneizaram-se as 5 porções dos peixes de cada lote.

As análises de composição química foram efetuadas em triplicata, conforme os seguintes protocolos: umidade, pelo método gravimétrico (estufa a $105^{\circ} \mathrm{C}$ ); cinzas pelo método gravimétrico, por calcinação (mufla a $550^{\circ} \mathrm{C}$ ); e proteínas pelo método de micro Kjeldahl (AOAC, 1997). Utilizou-se o fator N de 4,94 de acordo com Salo-Väänänen; Koivistoinen (1996). Os lipídeos totais foram determinados por extração com mistura de solventes a frio, conforme descrito por Bligh; Dyer (1959). O conteúdo de carboidratos foi dado pela diferença entre $100 \%$ e o somatório de umidade, cinzas, lipídeos e proteínas.

\subsection{Determinação do perfil de ácidos graxos}


O perfil de ácidos graxos foi determinado para os quatro lotes de cada uma das três espécies. Os lipídios foram esterificados segundo o estabelecido pela Comissão da Comunidade Européia (COMISSION DES COMMUNAUTÉS EUROPÉENES, 1977), todas as etapas do processo foram realizadas sob atmosfera de $\mathrm{N}_{2}$ gasoso. A separação dos ésteres metílicos foi realizada em um cromatógrafo a gás Varian, modelo CP-3380, equipado com detector de ionização de chama e coluna capilar de sílica fundida Varian modelo CP-Sil 88 (60m x 0,25mm; Cianopropil estabilizado). Os ácidos graxos foram identificados através da comparação dos tempos de retenção dos ésteres metílicos da amostra com os observados em uma amostra padrão (GLC - 68B e 68D, NuCheck). A quantificação dos ésteres metílicos foi realizada através da integração da área dos picos pelo software Star 6.0 (2003).

\subsection{Determinação da qualidade nutricional da fração lipídica}

Através dos dados do perfil lipídico foi determinada a qualidade nutricional da fração lipídica dos peixes estudados, através dos índices de aterogenicidade (IA) e trombogenicidade (IT) (ULBRICHT; SOUTHGATE, 1991) e da razão entre ácidos graxos hipocolesterolêmicos e hipercolesterolêmicos $\left(\mathrm{HH}=\sum\right.$ ác. graxos hipocolesterolêmicos/ $\sum$ ác. graxos hipercolesterolêmicos) (SANTOS-SILVA; BESSA; SANTOS-SILVA, 2002), os quais foram obtidos por meio das equações 1,2 e 3 .

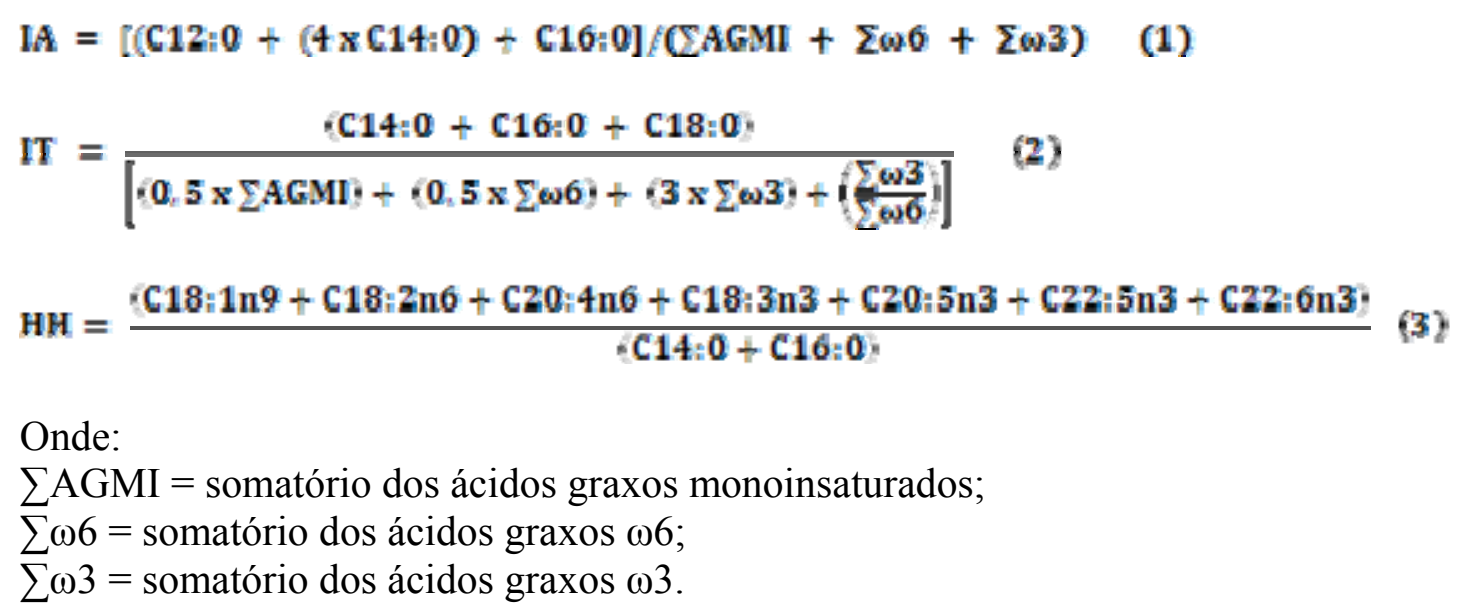

\subsection{Análise estatística}

Todos os resultados foram submetidos a tratamentos estatísticos utilizando-se o programa STATISTICA 7.0 (Stat Soft, Inc. 2004). Os resultados de caracterização física foram submetidos à análise de regressão linear múltipla, tendo como variável resposta o peso, encontrando-se o Coeficiente de Determinação $\left(\mathrm{R}^{2}\right)$ e Coeficiente de Correlação de Pearson $(\mathrm{R})$. Nas análises de composição química e perfil de ácidos graxos, a comparação entre as três espécies foi realizada 
através da Análise de Variância (ANOVA de um critério - teste F). Havendo diferenças entre as formulações complementou-se a análise com o teste Tukey. As análises estatísticas foram processadas ao nível alfa de $95 \%(\mathrm{p}<0,05)$.

\section{Resultados e Discussão}

\subsection{Caracterização física}

A Tabela 1 apresenta os resultados da análise biométrica das três espécies estudadas. E através da Figura 1 podem-se observar os gráficos e modelos matemáticos para o peso em função do comprimento zoológico e da circunferência corporal.

Tabela 1 - Caracterização Física

\begin{tabular}{|c|c|c|c|}
\hline Espécie & Peso (kg) & Comprimento (m) & Circunferência $(\mathrm{m})^{2}$ \\
\hline Gurijuba & $2,83 \pm 0,61$ & $0,65 \pm 0,04$ & $0,30 \pm 0,03$ \\
\hline Piramutaba & $1,85 \pm 0,96$ & $0,57 \pm 0,18$ & $0,26 \pm 0,09$ \\
\hline Dourada & $2,42 \pm 0,94$ & $0,62 \pm 0,09$ & $0,28 \pm 0,04$ \\
\hline
\end{tabular}

Figura 1 - Gráficos da análise de regressão linear múltipla para os parâmetros físicos avaliados (peso, comprimento e circunferência)

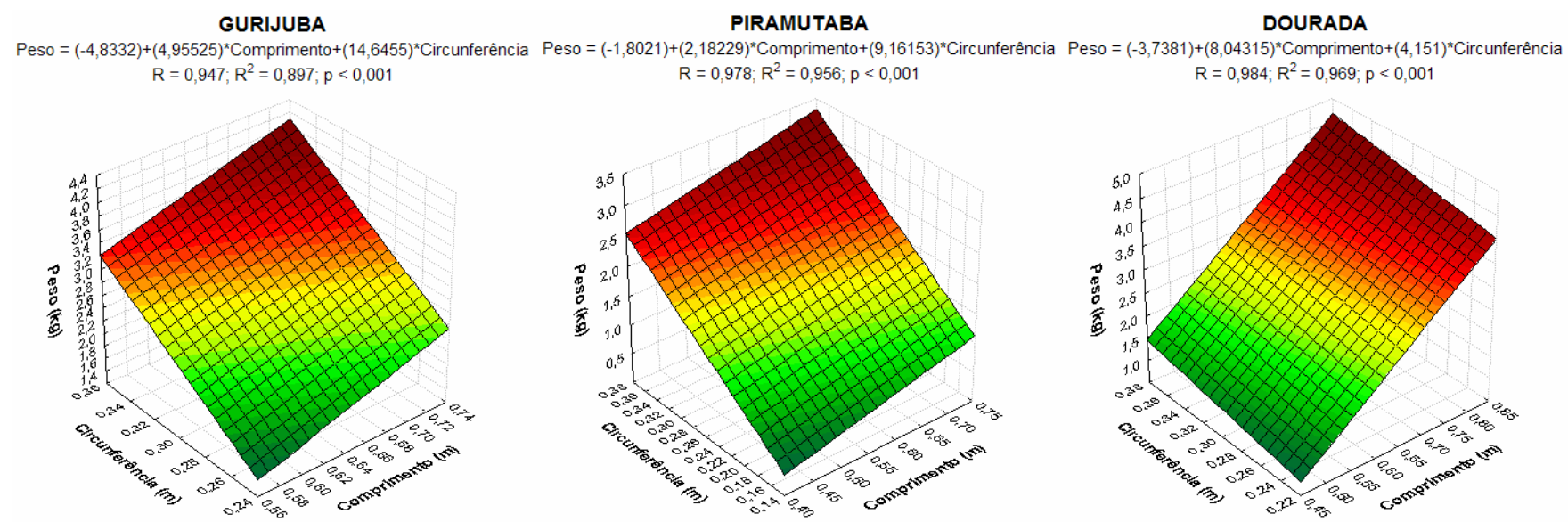

Os modelos gerados a partir da regressão linear múltipla apresentaram-se adequados para explicar o peso em função do comprimento e da circunferência nas três espécies, o que pode ser observado pelos elevados coeficientes de determinação $\left(\mathrm{R}^{2}>0,89\right)$, e que as variáveis, comprimento e circunferência estão fortemente relacionados ao peso, devidos os elevados valores de coeficiente de correlação (R). 


\subsection{Caracterização química}

Os resultados da composição química das três espécies constam na Tabela 2, onde se observa que a Gurijuba apresentou um teor significativamente maior de cinzas que as demais espécies. A Piramutaba apresentou um conteúdo de proteínas maior que a Gurijuba. E o teor lipídico da Dourada foi estatisticamente maior que o da Gurijuba, apesar disto, as três espécies estudadas podem, segundo Ackman (1989), ser considerados peixes magros, pois apresentaram teor lipídico inferior a $2 \%$.

Tabela 2 - Composição química (\%) das espécies estudadas ${ }^{1}$

\begin{tabular}{lccc}
\hline \multirow{2}{*}{\multicolumn{1}{c}{ Análise }} & \multicolumn{3}{c}{ Espécies } \\
\cline { 2 - 4 } & Gurijuba & Piramutaba & Dourada \\
\hline Umidade & $80,58 \pm 0,20$ & $80,30 \pm 0,28$ & $80,43 \pm 0,53$ \\
Cinzas & $1,12 \pm 0,04^{\mathrm{a}}$ & $1,01 \pm 0,03^{\mathrm{b}}$ & $0,97 \pm 0,00^{\mathrm{b}}$ \\
Proteínas & $15,47 \pm 0,42^{\mathrm{a}}$ & $18,47 \pm 0,79^{\mathrm{b}}$ & $16,80 \pm 0,90^{\mathrm{ab}}$ \\
Lipídeos & $0,37 \pm 0,01^{\mathrm{a}}$ & $0,43 \pm 0,04^{\mathrm{ab}}$ & $0,53 \pm 0,06^{\mathrm{b}}$ \\
Carboidratos & $2,46 \pm 0,36$ & - $^{\mathrm{b}}$ & $1,27 \pm 1,01$ \\
\hline Tetras diferentes indicam diferença significativa entre as espécies $(\mathrm{p}<0,05)$
\end{tabular}

\subsection{Determinação do perfil de ácidos graxos}

A Tabela 3 contem os resultados da análise cromatográfica da fração lipídica dos peixes. Foram identificados 9 ácidos graxos na Gurijuba e na Piramutaba e 12 ácidos na Dourada. Observase que para as três espécies o ácido graxo predominante foi o palmítico (16:0), seguido do DHA (22:6 - ácido docosahexaenóico) e do EPA (20:5 - ácido eicosapentaenóico).

Tabela 3 - Perfil de ácidos graxos ${ }^{1}$

\begin{tabular}{|c|c|c|c|c|}
\hline \multirow{2}{*}{$\begin{array}{l}\text { Ácido } \\
\text { graxo }\end{array}$} & \multirow{2}{*}{$\begin{array}{l}\text { Nome } \\
\text { Comum }\end{array}$} & \multicolumn{3}{|c|}{ Espécies } \\
\hline & & Gurijuba & Piramuba & Dourada \\
\hline C 14:0 & Mirístico & $2,50 \pm 0,17^{\mathrm{a}}$ & $5,31 \pm 0,48^{b}$ & $4,02 \pm 0,91^{\mathrm{ab}}$ \\
\hline C 14:1 n-5 & Miristoléico & - & - & $0,35 \pm 0,04$ \\
\hline C 16:0 & Palmítico & $27,51 \pm 0,01$ & $28,83 \pm 1,17$ & $29,14 \pm 1,76$ \\
\hline C 16:1 n-7 & Palmitoléico & $4,18 \pm 0,48^{\mathrm{a}}$ & $7,82 \pm 0,04^{b}$ & $4,94 \pm 0,87^{\mathrm{a}}$ \\
\hline C 18:0 & Esteárico & $6,81 \pm 0,71$ & $5,80 \pm 0,41$ & $6,78 \pm 0,16$ \\
\hline C 18:1 n-9 & Oléico & $10,86 \pm 0,13$ & $13,03 \pm 1,59$ & $11,97 \pm 0,34$ \\
\hline C 18:1 n-7 & vacénico & $3,40 \pm 0,24$ & $3,45 \pm 0,27$ & $3,44 \pm 0,21$ \\
\hline C 18:2 n-6 & Linoleico & - & - & $0,74 \pm 0,25$ \\
\hline C 22:1 n-9 & Erúcico & $12,22 \pm 0,89^{\mathrm{a}}$ & $5,98 \pm 0,55^{b}$ & $7,64 \pm 1,03^{b}$ \\
\hline C 20:5 n-3 & EPA & $12,63 \pm 0,45^{\mathrm{ab}}$ & $14,27 \pm 2,06^{\mathrm{a}}$ & $8,71 \pm 0,74^{\mathrm{b}}$ \\
\hline C 24:1 n-9 & Nervônico & - & - & $0,76 \pm 0,14$ \\
\hline C 22:6 n-3 & DHA & $19,91 \pm 0,13^{\mathrm{a}}$ & $15,54 \pm 0,39^{b}$ & $21,54 \pm 1,00^{\mathrm{a}}$ \\
\hline
\end{tabular}




\begin{tabular}{|c|c|c|c|}
\hline$\overline{\Sigma S F A s^{2}}$ & $36,82 \pm 0,87$ & $39,94 \pm 1,10$ & $39,93 \pm 1,00$ \\
\hline इMUFAs & $30,66 \pm 0,30$ & $30,27 \pm 1,34$ & $29,09 \pm 0,48$ \\
\hline ¿PUFAs & $32,54 \pm 0,57$ & $29,80 \pm 2,45$ & $30,98 \pm 1,48$ \\
\hline$\Sigma A G \omega 3$ & $32,54 \pm 0,57^{\mathrm{a}}$ & $29,80 \pm 2,45^{b}$ & $30,24 \pm 1,74^{a}$ \\
\hline$\Sigma A G \omega 6$ & - & - & $0,74 \pm 0,25$ \\
\hline
\end{tabular}

Letras diferentes indicam diferença significativa entre as espécies $(\mathrm{p}<0,05)$;

${ }^{2} \Sigma$ SFAs: Somatório dos ácidos graxos saturados; $\Sigma$ MUFAs: Somatório dos ácidos graxos monoinsaturados; $\Sigma$ PUFAs: Somatório dos ácidos graxos polinsaturados;

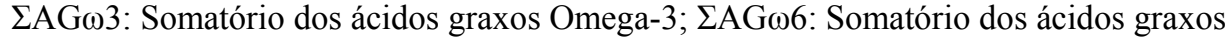

Omega-6.

Observa-se ainda que a Piramutaba apresentou maior teor de ácido mirístico (14:0) que a Gurijuba e maior teor de ácido palmitoléico (16:1) que as outras duas espécies estudadas. O conteúdo de ácido erúcico da Gurijuba foi superior ao encontrado na Piramutaba e na Dourada. Esta última apresentou teor significativamente menor de EPA que as demais espécies. A Piramutaba foi a espécie estudada com maior teor de DHA.

Com relação ao ácido graxo predominante por classe (saturado, monoinsaturado e polinsaturado), observou-se para os SFAs, o palmítico (16:0); para os MUFAs, o oléico (18:1) e para os PUFAs, o DHA, nas três espécies. Gutierrez; da Silva (1993) afirmam que peixes de água doce possuem maior conteúdo de ácidos graxos saturados que os de peixes marinhos, entretanto, no presente estudo, não foram observadas diferenças significativas entre o conteúdo do $\Sigma$ SFAs da gurijuba (peixe marinho) em relação à Piramutaba e à Dourada (peixes de água doce).

O conteúdo de EPA encontrado para a Piramutaba neste estudo foi $47 \%$ superior ao encontrado por Gutierrez; da Silva (1993), contudo, deve-se considerar que o conteúdo de gordura nos peixes pode variar amplamente entre as espécies e mesmo dentro de uma mesma espécie, dependendo da época do ano e de fatores, como sexo, grau de maturação das gônadas, idade e dieta (SIKORSKI; KOLAKOWSKA, 2002). O somatório de ácidos graxos $\omega 3$ foi significativamente menor na Piramutaba que nas demais espécies.

As recomendações dietéticas preconizadas pela American Heart Association são de $300 \mathrm{mg}$ a $500 \mathrm{mg}$ de EPA + DHA por dia, o que corresponde às seguintes porções de filé cru: $248 \mathrm{~g}$ de Gurijuba, 234 g de Piramutaba e 187 g de Dourada (KRAUSS et al, 2000; KRIS-ETHERTON; HARRIS; APPEL, 2002).

\subsection{Qualidade nutricional da fração lipídica}

Os índices de aterogenicidade (IA) e trombogenicidade (IT) indicam o potencial de estímulo à agregação plaquetária, isto é, quanto menores os valores de IA e IT, maior é quantidade de ácidos graxos anti-aterogênicos presentes em determinado óleo/gordura e consequentemente, maior é o potencial de prevenção ao aparecimento de doenças coronarianas (TURAN et al., 2007). 
Apesar de os ácidos graxos saturados, possuírem a característica de elevar os níveis de LDLcolesterol e reduzir os níveis de HDL-colesterol (o que contribui para a elevação dos riscos de doença coronariana), estes ácidos, no entanto, não apresentam o mesmo efeito hipercolesterolêmico, e dentre estes os que apresentam maior poder hipercolesterolêmico ou aterogênico são os ácidos mirístico (14:0), palmítico (16:0) e láurico (12:0), em ordem decrescente de atividade. O ácido esteárico (18:0), apesar de saturado, parece não possuir efeito sobre as lipoproteínas sanguíneas (MAHAN; ESCOTT-STUMP, 2005; DRISKELL, 2006).

A Tabela 4 apresenta os resultados da qualidade nutricional da fração lipídica das espécies estudadas. Não foi possível estabelecer o ratio $\Sigma \mathrm{AG} \omega 6 / \Sigma \mathrm{AG} \omega 3$ para a Gurijuba e para a Piramutaba, pois não foram detectados ácidos graxos n-6 nestas espécies. Entretanto, para a Dourada esta razão foi de 1:50 $(0,02)$, o que está muito acima do habitualmente consumido no ocidente, que é de 10-20:1 ao invés de 1-4:1, que é o recomendado (SIMOPOULOS, 1999).

Tabela 4 - Qualidade nutricional da fração lipídica das espécies estudadas ${ }^{1}$

\begin{tabular}{|c|c|c|c|}
\hline \multirow{2}{*}{ Ratio/ Índice } & \multicolumn{3}{|c|}{ Espécies } \\
\hline & Gurijuba & Piramutaba & Dourada \\
\hline$\Sigma A G \omega 6 / \Sigma A G \omega 3$ & - & - & $0,02 \pm 0,01$ \\
\hline 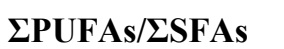 & $1,06 \pm 0,01$ & $0,99 \pm 0,12$ & $1,07 \pm 0,07$ \\
\hline IA & $0,59 \pm 0,02$ & $0,83 \pm 0,00$ & $0,64 \pm 0,14$ \\
\hline IT & $0,33 \pm 0,01$ & $0,38 \pm 0,03$ & $0,27 \pm 0,05$ \\
\hline HH & $1,08 \pm 0,02$ & $0,87 \pm 0,09$ & $0,94 \pm 0,07$ \\
\hline
\end{tabular}

Não houve diferença entre o ratio $\Sigma$ PUFAs/ $\Sigma$ SFAs das espécies estudadas, mas todas estão acima do recomendado pelo "Department of Health and Social Security" da Inglaterra (1984), segundo o qual a razão PUFAs/SFAs, não deve ser inferior a 0,45 , que se traduz uma dieta pouco saudável, principalmente em relação às doenças cardiovasculares.

Não foram observadas diferenças significativas entre os índices IA e IT nas espécies analisadas. Ramos Filho et al (2008) determinou os índices (IA e IT) em 4 espécies da região pantaneira e obteve os seguintes resultados, 0,54 e 0,59 para o cachara (Pseudoplatystoma fasciatum); 0,49 e 0,33 para o pintado (Pseudoplatystoma coruscans); 0,86 e 1,16 para o pacu (Piaractus mesopotamicus) e 0,70 e 0,35 para o dourado (Salminus maxillosus).

Rueda et al (2001) determinou IA e IT para o sargo-picudo (Diplodus puntazzo - Cetti, 1777), encontrando 0,51 e 0,24 , respectivamente para o peixe criado em cativeiro e 0,53 e 0,35 para o selvagem. Turan et al (2007) encontrou para raia-lenga (Raja clavata, L. 1758), 2,37 e 0,63 respectivamente.

Inversamente aos índices de aterogenicidade e trombogenicidade, para a razão entre ácidos graxos hipocolesterolêmicos e hipercolesterolêmicos $(\mathrm{HH})$, quanto maior o índice HH, mais 
adequado nutricionalmente é o óleo ou a gordura. Neste estudo, o valor de $\mathrm{HH}$ não diferiu entre os peixes. Ramos Filho et al (2008) encontrou os seguintes resultados, 1,75 para o cachara (Pseudoplatystoma fasciatum); 1,84 para o pintado (Pseudoplatystoma coruscans); 1,66 para o pacu (Piaractus mesopotamicus) e 1,49 para o dourado (Salminus maxillosus).

\begin{abstract}
The Pará state is the second major Brazilian fishing producing; however there is little nutritional information about amazonical fish species. In this study, the purpose was to characterize physically and chemically, determine the lipid profile and evaluate the species nutritional quality of the species: Gurijuba (Arius parkeri), Piramutaba (Brachyplastystoma vaillantii) and Dourada (Brachyplastystoma flavicans). The mathematical models of weight in function of length and circunference were suitable $\left(\mathrm{R}^{2}>0,89\right)$. The Gurijuba ash content $(1,12 \%)$ was greater than the others species. The Piramutaba proteins content $(18,47 \%)$ and the Dourada lipid content $(0,53 \%)$, were higher compared with Gurijuba; there were no differences between Piramutaba and Dourada in these aspects. The major fatty acid in the three species was the palmitic $(27,51$ to $29,14 \%)$, followed by DHA (15,54 to 21,54\%) and by EPA (8,71 to 14,27\%). The Piramutaba showed the lower total $\omega 3$ fatty acids $(29,8 \%)$. $\omega 6$ fatty acids were detected neither in Gurijuba nor in Piramutaba. The American Heart Association recommendations for EPA + DHA are attend by the follow filet crude portions: $248 \mathrm{~g}$ of Gurijuba, $234 \mathrm{~g}$ of Piramutaba e $187 \mathrm{~g}$ of Dourada. The Dourada showed excellent ratio $\Sigma A G \omega 6 / \Sigma A G \omega 3$ (1:50). All of species showed high nutritional quality in the lipid fraction, mean by low IA and IT index and high HH and ratio $\Sigma$ PUFAs $/ \Sigma$ SFAs.
\end{abstract}

Key-words: amazon fish; lipid profile, nutritional quality.

\title{
Referências
}

ACKMAN, R.G. Nutritional composition of fats in seafoods. Progress in Food and Nutrition Science. n. 13, p. 161$241,1989$.

AOAC - ASSOCIATION OF OFFICIAL ANALYTICAL CHEMISTS. Official methods of analysis of AOAC International. 16 ed. Gaitheersburg: AOAC, 1997.

BLIGH, E.G; DYER, W.J. A rapid method of total lipid extraction and purification. Canadian Journal Biochemistry Physiology, Ottawa, n.37, p.911-917, 1959.

BRASIL. Instituto Brasileiro do Meio Ambiente e dos Recursos Naturais Renováveis. Estatística da pesca 2006 Brasil: grandes regiões e unidades da federação. Brasília: IBAMA, 2008. 174 p.

COMISSION DES COMMUNAUTÉS EUROPÉENNES. Méthode d'analyse communautaire à utiliser pour la determination de la teneur en acid érucique, en ce qui concerne les graines prises en charge par les organismes d'intervention. Official Journal of the European Communities. L12, p.12-18, 1977.

DEPARTMENT OF HEALTH AND SOCIAL SECURITY. Diet and cardiovascular disease. Report on Health and Social Subjects, n. 28. London: HMSO, 1984.

DRISKELL, J.A. (ed.). Sports nutrition: fats and proteins. Boca Raton: CRC Press, 2006. 383 p.

DULLEMEIJER, C.; DURGA, J.; BROUWER, I.A.; VAN DE REST, O.; KOK, F.J.; BRUMMER, R-J.M.; VAN BOXTEL, M.P.J.; VERHOEF, P. n-3 Fatty acid proportions in plasma and cognitive performance in older adults. American Journal of Clinical Nutrition, New York, v. 86, n. 5, p. 1479-1485, November, 2007. 
GOLDING, J.; STEER, C.; EMMETT, P.; DAVIS, J.M.; HIBBELN, J.R. High levels of depressive symptoms in pregnancy with low omega-3 fatty acid intake from fish. Epidemiology, Baltimore, v. 20, n. 4, July, 2009.

GUTIERREZ, L.E.; DA SILVA, R.C.M. Fatty acid composition of commercially important fish from Brazil. Scientia Agricola. v. 50, n. 3, 1993.

ISO, H.; KOBAYASHI, M.; ISHIHARA, J.; SASAKI, S.; OKADA, K.; KITA, Y.; KOKUBO, Y.; TSUGANE, S. Intake of Fish and n3 Fatty Acids and Risk of Coronary Heart Disease Among Japanese. Circulation, Baltimore v. 113, p.195-202, 2006.

KRAUSS, R.M., ECKEL, R.H.; HOWARD, B.; APPEL, L.J.; DANIELS, S.R.; DECKELBAUM, R.J.; ERDMAN, J.W.; KRIS-ETHERTON,P.; GOLDBERG, I.J.; KOTCHEN, T.A.; LICHTENSTEIN, A.H.; MITCH, W.E.; MULLIS, R.; ROBINSON, K.; WYLIE-ROSETT, J.; ST. JEOR, S.; SUTTIE, J.; TRIBBLE, D.L.; BAZZARRE, T.L. AHA Dietary Guidelines : Revision 2000: A Statement for Healthcare Professionals From the Nutrition Committee of the American Heart Association. Circulation, Baltimore v. 102, p. 2284-2299, 2000.

KRIS-ETHERTON, P.M.; HARRIS, W.S.; APPEL, L.J. Fish Consumption, Fish Oil, Omega-3 Fatty Acids, and Cardiovascular Disease. Circulation, Baltimore v. 106, p. 2747-2757, 2002.

LANDS, W.E.M. Fish, Omega-3 and Human Health. 2nd ed. Champaign: AOCS Press, 2005. 215 p.

LANKINEN, M.; SCHWAB, U.; ERKKILÄ, A.; SEPPÄNEN-LAAKSO, T.; HANNILA, M-L; MUSSALO, H.; LEHTO, S.; UUSITUPA, M.; GYLLING, H.; Oresic, M. Fatty fish intake decreases lipids related to inflammation and insulin signaling-A lipidomics approach. PLoS ONE, Cambridge, v. 4, n. 4, e5258, 2009.

LARSEN, E.P.; HELDBO, J.; JESPERSEN, C.M.; NIELSEN, J. Development of a standard for quality assessment on fish for human consumption. In: HUSS, H. H.; JACOBSEN, M.; LISTON, J. (Eds). Quality Assurance in the fish industry. Proceedings of an international conference. Copenhagen, 1991. p. 351-358.

MAHAN, L.K.; ESCOTT-STUMP, S. KRAUSE: Alimentos, nutrição \& dietoterapia. 11ª ed. São Paulo: Roca, 2005.

MOFFATT, R.J. STAMFORD, B. (ed.). Lipid metabolism and health. Boca Raton: CRC Press, 2005. 365 p.

MOLNAR, J. Nutrition and wound healing. Boca Raton: CRC Press, 2006. 360 p.

MOSTOFSKY, D.I.; YEHUDA, S.; SALEM, N. Fatty acids: physiological and behavioral functions. Totowa: Humana Press, 2001. 435 p.

MOZAFFARIAN, D. Fish and n-3 fatty acids for the prevention of fatal coronary heart disease and sudden cardiac death. American Journal of Clinical Nutrition. New York, v. 87, n. 6, p. 1991-1996, June, 2008.

NELSON, D.L; COX, M.L. Lehninger: Principles of biochemistry. 4th ed. New York: W. H. Freeman and Company, 2005 .

RAMOS FILHO, M.M.; RAMOS, M.I.L.; HIANE, P.A.; SOUZA, M.T. Perfil de quarto espécies da região pantaneira de Mato Grosso do Sul. Ciência e Tecnologia de Alimentos, Campinas, v. 28, n. 2. p. 361-365, abr.-jun., 2008.

RUEDA, F.M.; HERNÁNDEZ, M.D.; EGEA, M.A.; AGUADO, F.; GARCÍA, B.; MARTÍNEZ, F.J. Differences in tissue fatty acid composition between reared and wild sharpsnout sea bream, Diplodus puntazzo (Cetti, 1777). British Journal of Nutrition, Cambridge, n. 86. p. 617-622, 2001.

SACKHEIM, G.I.; LEHMAN, D.D. Química e bioquímica para ciências biomédicas. Barueri: Manole, 2001.

SALO-VÄÄNÄNEN, P. P.; KOIVISTOINEN, P. E. Determination of protein in foods: comparison of net protein and crude protein $(\mathrm{N} \times 6.25)$ values. Food Chemistry, London, v. 57, n. 1, p. 27-31, 1996.

SANTOS-SILVA. J.; BESSA, R.J.B.; SANTOS-SILVA, F. Effects of genotype, feeding system and slaughter weigt on the quality of light lambs. II. Fatty acid composition of meat. Livestock Production Science, Roma, v. 77, n. 2/3. p. 187-194, 2002.

SIKORSKI, Z.E., KOLAKOWSKA, A. (ed.). Chemical and functional properties of food lipids. Boca Raton: CRC Press, 2002. 356 p. 
SIMOPOULOS, A.P. Essential fatty acids in health and chronic disease. American Journal of Clinical Nutrition. v. 70, n. 3, p. 560-569, September, 1999.

STAR 6.0. Star chromatography workstation, Varian Inc. version 6.0, 2003.

STATISTICA 7. StatSoft, Inc. STATISTICA (data analysis software system), version 7, 2004.

TURAN, H.; SÖNMEZ, G.; KAYA, Y. Fatty acid profile and proximate composition of the thornback ray (Raja clavata, L. 1758) from the Sinop coast in the Black Sea. Journal of FisheriesSciences.com. v. 1. n. 2. p. 97-103, 2007.

ULBRICHT, T.L.V.; SOUTHGATE, D.A.T. Coronary heart disease: seven dietary factors. Lancet, London, v. 338, n. 8773, p. 985-992, 1991.

VANSCHOONBEEK, K.; FEIJGE, M.A.H.; PAQUAY, M.; ROSING, J.; SARIS, W.; KLUFT, C.; GIESEN, P.L.A.; DE MAAT, M.P.M.; HEEMSKERK, J.W.M. Fibrinogen level and thrombin generation variable hypocoagulant effect of fish oil intake in humans: modulation of fibrinogen level and thrombin generation. Arteriosclerosis, Thrombosis, and Vascular Biology, Hagerstown, v. 24, p. 1734-1740, 2004.

WEAVER, K.L.; IVESTER, P.; SEEDS, M.; CASE, L. D.; ARM, J.P.; CHILTON, F.H. Effect of dietary fatty acids on inflammatory gene expression in healthy humans. The Journal of Biological Chemistry, Bethesda, v. 284, n. 23, June 5, p. 15400-15407, 2009.

Nome completo: Ádria de Sousa Bentes

Filiação institucional: Universidade Federal do Pará - UFPA

Departamento: Departamento de Engenharia Química e de Alimentos; Departamento de Nutrição Função ou cargo ocupado: Mestranda em ciência e tecnologia de alimentos e graduanda em Nutrição

Titulação: Graduada em Tecnologia Agroindustrial - Alimentos

Endereço completo para correspondência:

Tv. Barão do Triunfo 1807, Vila Aleixo, casa 01, Bairro Pedreira, CEP 66080-550, Belém, Pará, Brasil.

Telefone para contato: (91) 3236-1187

e-mail: adriabentes@ufpa.br

Nome completo: Hugo Antonio Lima de Souza

Filiação institucional: Universidade Federal do Pará - UFPA

Departamento: Departamento de Engenharia Química e de Alimentos

Função ou cargo ocupado: Mestrando em ciência e tecnologia de alimentos

Titulação: Graduado em Tecnologia Agroindustrial - Alimentos

Endereço completo para correspondência:

Tv. Castelo Branco 1116, apto 1403, Bairro São Brás, CEP 66063-000, Belém, Pará, Brasil.

Telefone para contato: (91) 3249-3236

e-mail: hugosouza@ufpa.br 
Nome completo: Xaene Maria Fernandes Duarte Mendonça

Filiação institucional: Universidade Federal do Pará - UFPA

Departamento: Departamento de Nutrição

Função ou cargo ocupado: Professora Adjunto I da Faculdade de Nutrição da UFPA

Titulação: Doutora em Tecnologia de Processos Químicos e Bioquímicos

Endereço completo para correspondência:

Faculdade de Nutrição - FANUT, Av. Generalíssimo Deodoro 01, Bairro Umarizal, CEP 66050060, Belém, Pará, Brasil.

Telefone para contato: (91) 3201-6850

e-mail:xaene@hotmail.com

Nome completo: Marilda Garcia Simões

Filiação institucional: Universidade do Estado do Pará - UEPA

Departamento: Departamento de Tecnologia de Alimentos

Função ou cargo ocupado: Professora Assistente II do Curso de Tecnologia Agroindustrial Ênfase em Alimentos

Titulação: Mestre em Alimentos e Nutrição

Endereço completo para correspondência:

Centro de Ciência Naturais e Tecnologia - CCNT, Tv. Éneas Pinheiro 2626, Bairro Marco, CEP 66095-100, Belém, Pará, Brasil.

Telefone para contato: (91) 3276-9511, Ramal: 204

e-mail: marildags@uol.com.br 